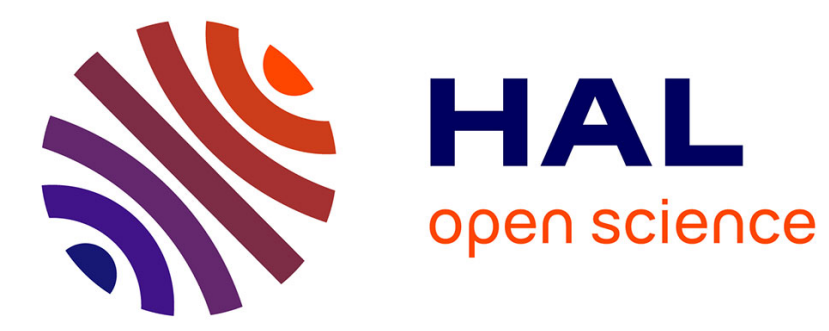

\title{
STM systems: Enforcing strong isolation between transactions and non-transactional code
}

Tyler Crain, Eleni Kanellou, Michel Raynal

\section{To cite this version:}

Tyler Crain, Eleni Kanellou, Michel Raynal. STM systems: Enforcing strong isolation between transactions and non-transactional code. [Research Report] RR-7970, INRIA. 2012. hal-00699903

\section{HAL Id: hal-00699903 \\ https://hal.inria.fr/hal-00699903}

Submitted on 21 May 2012

HAL is a multi-disciplinary open access archive for the deposit and dissemination of scientific research documents, whether they are published or not. The documents may come from teaching and research institutions in France or abroad, or from public or private research centers.
L'archive ouverte pluridisciplinaire HAL, est destinée au dépôt et à la diffusion de documents scientifiques de niveau recherche, publiés ou non, émanant des établissements d'enseignement et de recherche français ou étrangers, des laboratoires publics ou privés. 
STM systems: Enforcing strong isolation between transactions and non-transactional code

Tyler CRAIN, Eleni KANELLOU, Michel RAYNAL tyler.crain@irisa.fr, eleni.kanellou@irisa.fr,raynal@irisa.fr

RESEARCH 



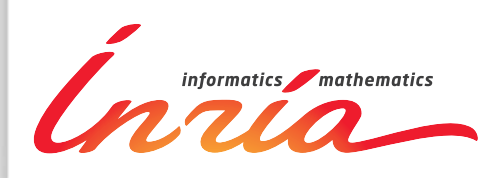

\title{
STM systems: Enforcing strong isolation between transactions and non-transactional code
}

\author{
Tyler CRAIN*, Eleni KANELLOU*, Michel RAYNAL $\dagger^{*}$ \\ tyler.crain@irisa.fr,eleni.kanellou@irisa.fr,raynal@irisa.fr \\ Project-Team ASAP
}

Research Report $n^{\circ} 7970$ - May 2012 - 15 pages

\begin{abstract}
Transactional memory (TM) systems implement the concept of an atomic execution unit called transaction in order to discharge programmers from explicit synchronization management. But when shared data is atomically accessed by both transaction and non-transactional code, a TM system must provide strong isolation in order to overcome consistency problems. Strong isolation enforces ordering between non-transactional operations and transactions and preserves the atomicity of a transaction even with respect to non-transactional code. This paper presents a TM algorithm that implements strong isolation with the following features: (a) concurrency control of non-transactional operations is not based on locks and is particularly efficient, and (b) any non-transactional read or write operation always terminates (there is no notion of commit/abort associated with them).
\end{abstract}

Key-words: Transactional Memory, Strong Isolation, Atomicity

* IRISA, Université de Rennes 35042 Rennes Cedex, France

$\dagger$ Institut Universitaire de France

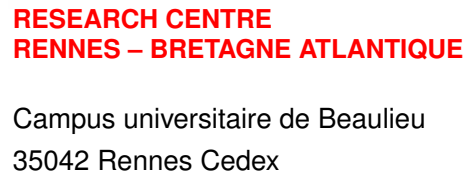




\section{Mémoire Transactionnelle: Imposer l'isolement forte entre transactions et opérations non-transactionnelle}

Résumé : Ce rapport présente un algorithme de mémoire transactionnelle qui impose l'isolement forte entre transactions et opérations non-transactionnelle.

Mots-clés : mémoire transactionnelle, atomicité 


\section{Introduction}

STM Systems. Transactional Memory (TM) [8, 17] has emerged as an attempt to allow concurrent programming based on sequential reasoning: By using TM, a user should be able to write a correct concurrent application, provided she can create a correct sequential program. The underlying TM system takes care of the correct implementation of concurrency. However, while most existing TM algorithms consider applications where shared memory will be accessed solely by code enclosed in a transaction, it still seems imperative to examine the possibility that memory is accessed both inside and outside of transactions.

Strong vs Weak Isolation. TM has to guarantee that transactions will be isolated from each other, but when it comes to transactions and non-transactional operations, there are two paths a TM system can follow: it may either act oblivious to the concurrency between transactions and non-transactional operations, or it may take this concurrency into account and attempt to provide isolation guarantees even between transactional and nontransactional operations. The first case is referred to as weak isolation while the second case is referred to as strong isolation. (This distinction of guarantees was originally made in [11], where reference was made to "weak atomicity" versus "strong atomicity".)

While weak isolation violates the isolation principle of the transaction abstraction, it could nevertheless be anticipated and used appropriately by the programmer, still resulting in correctly functioning applications. This would require the programmer to be conscious of eventual race conditions between transactional and nontransactional code that can change depending on the STM system used.

Desirable Properties. In order to keep consistent with the spirit of TM principles, however, a system should prevent unexpected results from occurring in presence of race conditions. Furthermore, concurrency control should ideally be implicit and never be delegated to the programmer [2, 12]. These are the reasons for which strong isolation is desirable. Under strong isolation, the aforementioned scenarios, where non-transactional operations violate transaction isolation, would not be allowed to happen. An intuitive approach to achieving strong isolation is to treat each non-transactional operation that accesses shared data as a "mini-transaction", i.e., one that contains a single operation. In that case, transactions will have to be consistent (see Sect. 27) not only with respect to each other, but also with respect to the non-transactional operations. However, while the concept of the memory transaction includes the possibility of abort, the concept of the non-transactional operation does not. This means that a programmer expects that a transaction might fail, either by blocking or by aborting. Non-transactional accesses to shared data, though, will usually be read or write operations, which the programmer expects to be atomic. While executing, a read or write operation is not expected to be de-scheduled, blocked or aborted.

Content of the Paper. This paper presents a TM algorithm which takes the previous issues into account. It is built on top of TM algorithm TL2 [5], a word-based TM algorithm that uses locks. More precisely, TL2 is modified to provide strong isolation with non-transactional read and write operations. However, the algorithm is designed without the use of locks for non-transactional code, in order to guarantee that their execution will always terminate. To achieve this, two additional functions are specified, which substitute conventional read or write operations that have to be performed outside of a transaction. Possible violations of correctness under strong isolation are reviewed in Sect. 2. The TL2 algorithm is described in Sect. 3 . Section 4 describes the proposed algorithm that implements strong isolation for TL2, while Sect. 5 concludes the paper by summarizing the work and examining possible applications.

\section{Correctness and Strong Isolation}

Consistency Issues. Commonly, consistency conditions for TM build on the concept of serializability [14], a condition first established for the study of database transactions.

A concurrent execution of transactions is serializable, if there exists a serialization, i.e., a legal sequential execution equivalent to it. Serializability refers only to committed transactions, however, and fails to take into account the possible program exceptions that a TM transaction may cause - even if it aborts - when it observes an inconsistent state of memory.

Opacity [6], a stricter consistency condition for TM, requires that both committed as well as aborted transactions observe a consistent state of shared memory. This implies that in order for a concurrent execution of memory 
transactions to be opaque, there must exist an equivalent, legal sequential execution that includes both committed transactions and aborted transactions, albeit reduced to their read prefix. Other consistency conditions have also been proposed, such as virtual world consistency [9]. It is weaker than opacity while keeping its spirit (i.e., it depends on both committed transactions and aborted transactions).

Transaction vs Non-transactional Code. In a concurrent environment, shared memory may occasionally be accessed by both transactions as well as non-transactional operations. Traditionally, however, transactions are designed to synchronize only with other transactions without considering the possibility of non-transactional code; a program that accesses the same shared memory both transactionally and non-transactionally would be considered incorrect. A TM system that implements opacity minimally guarantees consistency between transactional accesses, however, consistency violations may still be possible in the presence of concurrent non-transactional code. Given this, it can still be acceptable to have concurrent environments that may be prone to some types of violations, as is the case with systems that provide weak isolation [11,18]. Under weak isolation, transactional and non-transactional operations can be concurrent, but the programmer has to be aware of how to handle these. Interestingly, this possibility of co-existence of two different paradigms between strong and weak isolation reveals two different interpretations of transactional memory: On one hand considering TM as an implementation of shared memory, and, on the other hand, considering TM as an additional way of achieving synchronization, to be used alongside with locks, fences, and other traditional methods.

Under weak isolation, transactions are considered to happen atomically only with respect to other transactions. It is possible for non-transactional operations to see intermediate results of transactions that are still live. Conversely, a transaction may see the results of non-transactional operations that happened during the transaction's execution. If this behavior is not considered acceptable for an application, then the responsibility to prevent it is delegated to the programmer of concurrent applications for this system. However, in order to spare the programmer this responsibility, both the transactional memory algorithm as well as the non-transactional read and write operations must be implemented in a way that takes their co-existence into account. Such an implementation that provides synchronization between transactional and non-transactional code is said to provide strong isolation.

Providing Strong Isolation. There are different definitions in literature for strong isolation [7, 10, 11]. In this paper we consider strong isolation to be the following: (a) non-transactional operations are considered as "mini" transactions which never abort and contain only a single read or write operation, and (b) the consistency condition for transactions is opacity.

This definition implies that the properties that are referred to as containment and non-interference [11] are satisfied. Containment is illustrated in the left part of Fig. 1. There, under strong isolation, we have to assume that transaction $T_{1}$ happens atomically, i.e.,"all or nothing", also with respect to non-transactional operations. Then, while $T_{1}$ is alive, no non-transactional read, such as $R_{x}$, should be able to obtain the value written to $x$ by $T_{1}$. Non-interference is illustrated in the right part of Fig. 1. Under strong isolation, non-transactional code should not interfere with operations that happen inside a transaction. Therefore, transaction $T_{1}$ should not be able to observe the effects of operations $W_{x}$ and $W_{y}$, given that they happen concurrently with it, while no opacity-preserving serialization of $T_{1}, W_{x}$ and $W_{y}$ can be found. Non-interference violations can be caused, for example, by nontransactional operations that are such as to cause the ABA problem for a transaction that has read a shared variable $x$. An additional feature of strong isolation, implemented in this paper, is that non-transactional read and write operations never block or abort. For this reason, it is termed terminating strong isolation.
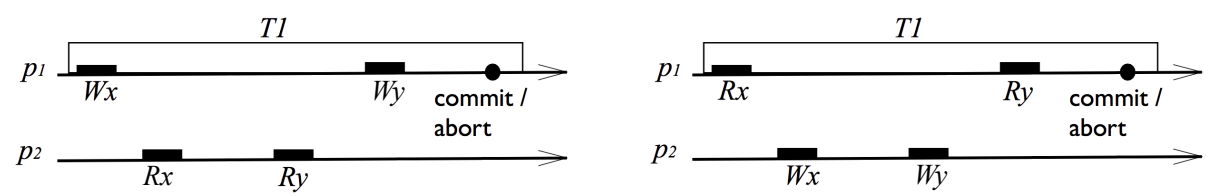

Figure 1: Left: Containment (operation $R_{x}$ should not return the value written to $x$ inside the transaction). Right: Non-Interference (wile it is still executing, transaction $T_{1}$ should not have access to the values that were written to $x$ and $y$ by process $\left.p_{2}\right)$.

Privatization/Publication. A discussion of the co-existence of transactional and non-transactional code would not be complete without mentioning the privatization problem. An area of shared memory is privatized, when 
a process that modified it makes it inaccessible to other concurrent processes 1 with the purpose being that the process can then access the memory without using synchronization operations [20]. A typical example of privatization would be the manipulation of a shared linked list. The removal of a node by a transaction $T_{i}$, for private use, through non-transactional code, by the process that invoked $T_{i}$, constitutes privatization. Then, $T_{i}$ is called privatizing transaction. While the privatization is not visible to all processes, inconsistencies may arise, given that for $T_{i}$ 's process the node is private but for other processes, the node is still seen as shared. Several solutions have been proposed for the privatization problem such as [1, 4, 16]. A system that provides strong isolation has the advantage of inherently also solving the privatization problem, because it inherently imposes synchronization between transactional and non-transactional code.

\section{A Brief Presentation of TL2}

TL2, aspects of which are used in this paper, has been introduced by Dice, Shalev and Shavit in 2006 [5]. The word-based version of the algorithm is used, where transactional reads and writes are to single memory words.

Main Features of TL2. The shared variables that a transaction reads form its read set, while the variables it updates form the write set. Read operations in TL2 are invisible, meaning that when a transaction reads a shared variable, there is no indication of the read to other transactions. Write operations are deferred, meaning that TL2 does not perform the updates as soon as it "encounters" the shared variables that it has to write to. Instead, the updates it has to perform are logged into a local list (also called redo log) and are applied to the shared memory only once the transaction is certain to commit. Read-only transactions in TL2 are considered efficient, because they don't need to maintain local copies of a read or write set and because they need no final read set validation in order to commit. To control transaction synchronization, TL2 employs locks and logical dates.

Locks and Logical Date. A lock is associated with each shared variable. When a transaction attempts to commit it first has to obtain the locks of the variables of its write set, before it can update them. Furthermore, a transaction has to check the logical dates of the variables in its read set in order to ensure that the values it has read correspond to a consistent snapshot of shared memory. TL2 implements logical time as an integer counter denoted GVC. When a transaction starts it reads the current value of $G V C$ into local variable, $r v$. When a transaction attempts to commit, it performs an increment-and-fetch on $G V C$, and stores the return value in local variable $w v$ (which can be seen as a write version number or a version timestamp). Should the transaction commit, it will assign its $w v$ as the new logical date of the shared variables in its write set. A transaction must abort if its read set is not valid. Its read set is valid if the logical date of every item in the set is less than the transaction's $r v$ value. If, on the contrary, the logical date of a read set item is larger than the $r v$ of the transaction, then a concurrent transaction has updated this item, invalidating the read.

\section{Implementing Terminating Strong Isolation}

A possible solution to the problem of ensuring isolation in the presence of non-transactional code consists in using locks: Each shared variable would then be associated with a lock and both transactions as well as non-transactional operations would have to access the lock before accessing the variable.

Locks are already used in TM algorithms - such as TL2 itself - where it is however assumed that shared memory is only accessed through transactions. The use of locks in a TM algorithm entails blocking and may even lead a process to starvation. However, it can be argued that these characteristics are acceptable, given that the programmer accepts the fact that a transaction has a duration and that it may even fail: The fact that there is always a possibility that a transaction will abort means that the eventuality of failure to complete can be considered a part of the transaction concept.

On the contrary, when it comes to single read or write accesses to a shared variable, a non-transactional operation is understood as an event that happens atomically and completes. Unfortunately strong isolation implemented with locks entails the blocking of non-transactional read and write operations and would not provide termination.

Given that this approach would be rather counter-intuitive for the programmer (as well as possibly detrimental for program efficiency), the algorithm presented in this section provides a solution for adding strong isolation

\footnotetext{
${ }^{1}$ Conversely, a memory area is made public when it goes from being exclusively accessible by one process to being accessible by several processes [19]. This is referred to as the publication problem and the consistency issues that arise are analogous.
} 
which is not based on locks for the execution of non-transactional operations. This algorithm builds on the base of TM algorithm TL2 and extends it in order to account for non-transactional operations. While read and write operations that appear inside a transaction follow the original TL2 algorithm rather closely (cheap read only transactions, commit-time locking, write-back), the proposed algorithm specifies non-transactional read and write operations that are to be used by the programmer, substituting conventional shared memory read and write operations. TM with strong isolation has also been proposed in software [15, 18] in hardware [13], and has been suggested to be too costly [3]. This work differs from other implementations in that it is terminating and is implemented on top of a state-of-the-art STM in order to avoid too much extra cost.

\subsection{Memory Set-up and Data Structures.}

Memory Set-up. The underlying memory system is made up of atomic read/write registers. Moreover some of them can also be accessed by the the following two operations. The operation denoted Fetch\&increment() atomically adds one to the register and returns its previous value. The operation denoted $\mathrm{C} \& \mathrm{~S}()$ (for compare and swap) is a conditional write. $\mathrm{C} \& \mathrm{~S}(x, a, b)$ writes $b$ into $x$ iff $x=a$. In that case it returns true. Otherwise it returns false.

The proposed algorithm assumes that the variables are of types and values that can be stored in a memory word. This assumption aids in the clarity of the algorithm description but it is also justified by the fact that the algorithm extends TL2, an algorithm that is designed to be word-based.

As in TL2, the variable $G V C$ acts as global clock which is incremented by update transactions. Apart from a global notion of "time", there exists also a local one; each process maintains a local variable denoted time, which is used in order to keep track of when, with respect to the $G V C$, a non-transactional operation or a transaction was last performed by the process. This variable is then used during non-transactional operations to ensure the (strict) serialization of operations is not violated.

In TL2 a shared array of locks is maintained and each shared memory word is associated with a lock in this array by some function. Given this, a memory word directly contains the value of the variable that is stored in it. Instead, the algorithm presented here, uses a different memory set-up that does not require a lock array, but does require an extra level of indirection when loading and storing values in memory. Instead of storing the value of a variable directly to a memory word, each write operation on variable var, transactional or non-transactional, first creates an algorithm-specific structure that contains the new value of var, as well as necessary meta-data and second stores a pointer to this structure in the memory word. The memory set-up is illustrated in Fig. 2, Given the particular memory arrangement that the algorithm uses, pointers are used in order to load and store items from memory. ${ }^{2}$

T-record and NT-record. These algorithm-specific data structures are shared and can be of either two kinds, which will be referred to as T-records and NT-records. A T-record is created by a transactional write operation while an NT-record is created by a non-transactional write operation.

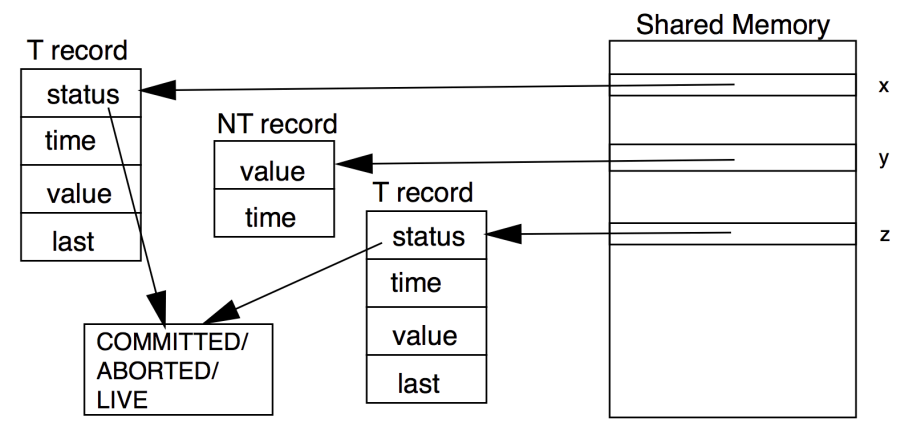

Figure 2: The memory set-up and the data structures that are used by the algorithm.

New T-records are created during the transactional write operations. Then during the commit operation the pointer stored at $a d d r$ is updated to point to this new T-record. During NT-write operations new NT-records are

\footnotetext{
${ }^{2}$ The following notation is used. If $p t$ is a pointer, $p t \downarrow$ is the object pointed to by $p t$. if $a a$ is an object, $\uparrow a a$ is a pointer to $a a$. Hence $((\uparrow a a) \downarrow=a a$ and $\uparrow(p t \downarrow)=p t$.
} 
created and the pointer at $a d d r$ is updated to point to the records.

When a read operation - be it transactional or non-transactional - accesses a shared variable it cannot know beforehand what type of record it will find. Therefore, it can be seen in the algorithm listings, that whenever a record is accessed, the operation checks its type, i.e., it checks whether it is a T-record or an NT-record (for example, line 02 in Fig. 3 contains such a check. A T-record is "of type T", while an NT-record is "of type NT").

T-record. A T-record is a structure containing the following fields.

status This field indicates the state of the transaction that created the T-record. The state can either be LIVE, COMMITTED or ABORTED. The state is initially set to LIVE and is not set to COMMITTED until during the commit operation when all locations of the transaction's write set have been set to point to the transaction's T-records and the transaction has validated its read set. Since a transaction can write to multiple locations, the status field does not directly store the state, instead it contains a pointer to a memory location containing the state for the transaction. Therefore the status field of each T-record created by the same transaction will point to the same location. This ensures that any change to the transaction's state is immediately recognized at each record.

time The time field of a T-record contains the value of the GVC at the moment the record was inserted to memory. This is similar to the logical dates of TL2.

value This field contains the value that is meant to be written to the chosen memory location.

last During the commit operation, locations are updated to point to the committing transaction's T-records, overwriting the previous value that was stored in this location. Failed validation or concurrent non-transactional operations may cause this transaction to abort after it updates some memory locations, but before it fully commits. Due to this, the previous value of the location needs to be available for future reads. Instead of rolling back old memory values, the last field of a T-record is used, storing the previous value of this location.

NT-record. An NT-record is a structure containing the following fields.

value This field contains the value that is meant to be written to the chosen memory location.

time As in the case of T-records, the time field of NT-records also stores the value of the GVC when the write took place.

Due to this different memory structure a shared lock array is no longer needed, instead of locking each location in the write set during the commit operation, this algorithm performs a compare and swap directly on each memory location changing the address to point to one of its T-records. After a successful compare and swap and before the transactions status has been set to COMMITTED or ABORTED, the transaction effectively owns the lock on this location. Like in TL2, any concurrent transaction that reads the location and sees that it is locked (status = LIVE) will abort itself.

Transactional Read and Write Sets. Like TL2, read only transactions do not use read sets while update transactions do. The read set is made up of a set of tuples for each location read, $\langle a d d r, v a l u e\rangle$ where $a d d r$ is the address of the location read and value is the value. The write set is also made up of tuples for each location written by the transaction, $\langle a d d r, i t e m\rangle$ where $a d d r$ is the location to be written and item is a T-record for this location.

\subsubsection{Discussion.}

One advantage of the TL2 algorithm is in its memory layout. This is because reads and writes happen directly to memory (without indirection) and the main amount of additional memory that is used is in the lock array. Unfortunately this algorithm breaks that and requires an additional level of indirection as well as additional memory per location. While garbage collection will be required for old T- and NT-records, here we assume automatic garbage collection such as that provided in Java, but additional solutions will be explored in future work. These additional requirements can be an acceptable trade-off given that they are only needed for memory that will be shared between transactions. In the appendix of this paper we present two variations of the algorithm that trade off different memory schemes for different costs to the transactional and non-transactional operations. 


\subsection{Description of the Algorithm.}

The main goal of the algorithm is to provide strong isolation in such a way that the non-transactional operations are never blocked. In order to achieve this, the algorithm delegates most of its concurrency control and consistency checks to the transactional code. Non-transactional operations access and modify memory locations without waiting for concurrent transactions and it is mainly up to transactions accessing the same location to deal with ensuring safe concurrency. As a result, this algorithm gives high priority to non-transactional code.

\subsection{Non-transactional Operations.}

Algorithm-specific read and write operations shown in Fig. 3 must be used when a shared variable is accessed accessed outside of a transaction. This be done by hand or applied by a complier.

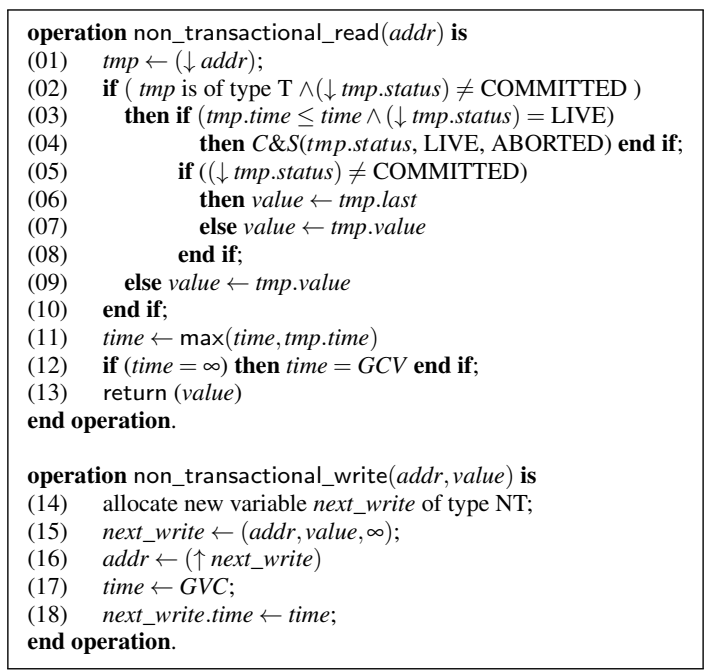

Figure 3: Non-transactional operations for reading and writing a variable.

Non-transactional Read. The operation non_transactional_read() is used to read, when not in a transaction, the value stored at $a d d r$. The operation first dereferences the pointer stored at $a d d r$ (line 01). If the item is a T-record that was created by a transaction which has not yet committed then the value field cannot be immediately be read as the transaction might still abort. Also if the current process has read (or written to) a value that is more recent then the transaction (meaning the process's time field is greater or equal to the T-records time, line 033 then the transaction must be directed to abort (line 04p so that opacity and strong isolation (containment specifically) is not violated. From a T-record with a transaction that is not committed, the value from the last field is stored to a local variable (line 06 and will be returned on operation completion. Otherwise the value field of the T- or NT-record is used (line 07).

Next the process local variable time is advanced to the maximal value among its current value and the logical date of the T- or NT-record whose value was read. Finally if time was set to $\infty$ on line 11 (meaning the T- or NTrecord had yet to set its time), then it is updated to the $G C V$ on line 12 . The updated time value is used to prevent consistency violations. Once these book-keeping operations are finished, the local variable value is returned (line 13).

Non-transactional Write. The operation non_transactional_write() is used to write to a shared variable var by non-transactional code. The operation takes as input the address of the shared variable as well as the value to be written to it. This operation creates a new NT-record (line 14], fills in its fields (line 15) and changes the pointer stored in $a d d r$ so that it references the new record it has created (line 16). Unlike update transactions, non-transactional writes do not increment the global clock variable $G C V$. Instead they just read $G C V$ and set the NT-record's time value as well as the process local time to the value read (line 17 and 18 . Since the GCV is not incremented, several NT-records might have the same time value as some transaction. When such a situation is recognized where a live transaction has the same time value as an NT-record the transaction must be aborted 
(if recognized during an NT-read operation, line 04) or perform read set validation (if during a transactional read operation, line 23 of Fig. 47. This is done in order to prevent consistency violations caused by the NT-writes not updating the $G C V$.

\subsection{Transactional Read and Write Operations.}

The transactional operations for performing reads and writes are presented in Fig. 4

Transactional Read. The operation transactional_read() takes $a d d r$ as input. It starts by checking whether the desired variable already exists in the transaction's write set, in which case the value stored there will be returned (line 19). If the variable is not contained in the write set, the pointer in $a d d r$ is dereferenced (line 20) and set to tmp. Once this is detected to be a T- or NT-record some checks are then performed in order to ensure correctness.

In the case that tmp is a T-record the operation must check to see if the status of the transaction for this record is still LIVE and if it is the current transaction is aborted (line 29]. This is similar to a transaction in TL2 aborting itself when a locked location is found. Next the T-record's time field is checked, and (similar to TL2) if it greater then the process's local $r v$ value the transaction must abort (line 32) in order to prevent consistency violations. If this succeeds without aborting then the local variable value is set depending on the stats of the transaction that created the T-record (line 29 30.

In case tmp is an NT-record (line 21, the operation checks whether the value of the time field is greater or equal to the process local $r v$ value. If it is, then this write has possibly occurred after the start of this transaction and there are several possibilities. In the case of an update transaction validation must be preformed, ensuring that none of the values it has read have been updated (line 23. In the case of a read only transaction, the transaction is aborted and restarted as an update transaction (line 24]. It is restarted as an update transaction so that it has a read set that it can validate in case this situation occurs again. Finally local variable value is set to be the value of the value field of the tmp (line 26).

It should be noted that the reason why the checks are performed differently for NT-records and T-records is because the NT-write operations do not update the global clock value while update transaction do. This means that the checks must be more conservative in order to ensure correctness. If performing per value validation or restarting the transaction as an update transaction is found to be too expensive, a third possibility would be to just increment the global clock, then restart the transaction as normal.

Finally to finish the read operation, the $\langle a d d r$, value $\rangle$ is added to the read set if the transaction is an update transaction (line 34), and the value of the local variable value is returned.

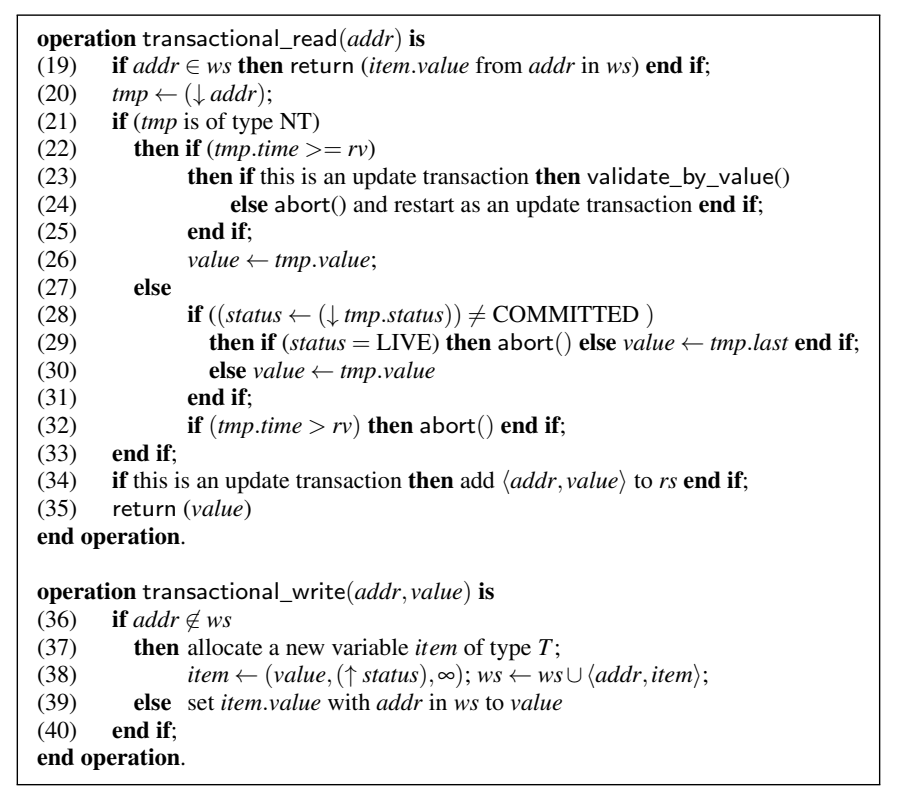

Figure 4: Transactional operations for reading and writing a variable. 
Transactional Write. The transactional_write() operation takes addr as input value, as well as the value to be written to var. As TL2, the algorithm performs commit-time updates of the variables it writes to. For this reason, the transactional write operation simply creates a T-record and fills in some of its fields (lines 37- 38) and adds it to the write set. However, in the case that a T-record corresponding to $a d d r$ was already present in the write set, the value field of the corresponding T-record is simply updated (line 39 .

Begin and End of a Transaction The operations that begin and end a transaction are begin_transaction( () and try_to_commit(), presented in Fig. 5] Local variables necessary for transaction execution are initialized by begin_transaction(). This includes $r v$ which is set to $G C V$ and, like in TL2, is used during transactional reads to ensure correctness, as well as status which is set to LIVE and the read and write sets which are initialized as empty sets. (lines 41,43 ,

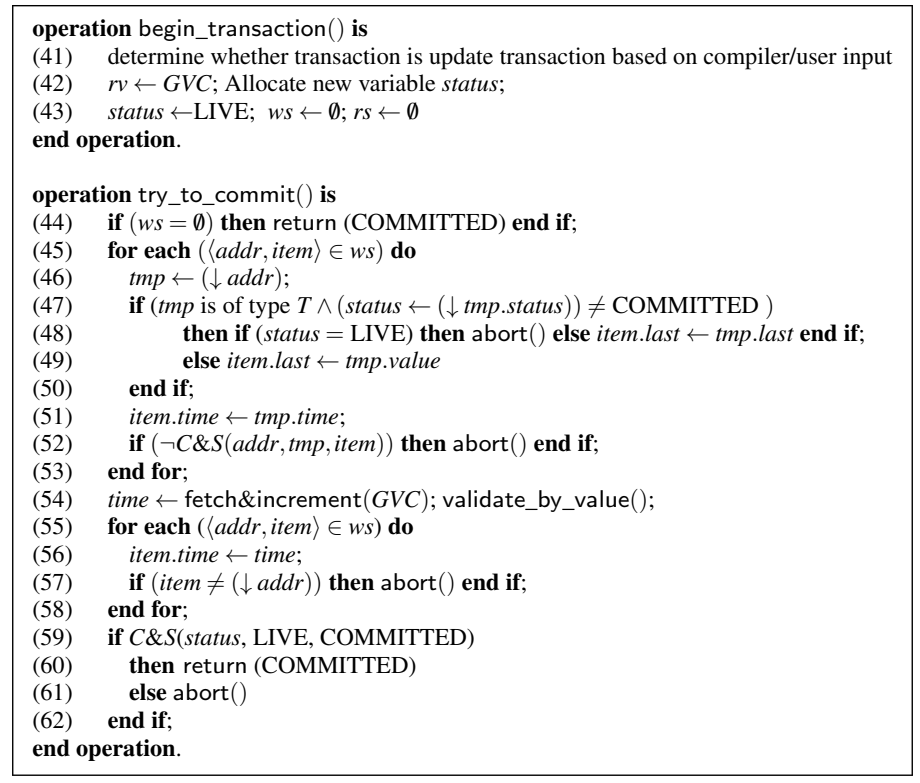

Figure 5: Transaction begin/commit.

After performing all required read and write operations, a transaction tries to commit, using the operation try_to_commit(). Similar to TL2, a try_to_commit() operation starts by trivially committing if the transaction was a read-only one (line 44p while an update transaction must announce to concurrent operations what locations it will be updating (the items in the write set). However, the algorithm differs here from TL2, given that it is faced with concurrent non-transactional operations that do not rely on locks and never block. This implies that even after acquiring the locks for all items in its write set, a transaction could be "outrun" by a non-transactional operation that writes to one of those items causing the transaction to be required to abort in order to ensure correctness. As described previously, while TL2 locks items in its write set using a lock array, this algorithm compare and swaps pointers directly to the T-records in its write set (lines 45 53) while keeping a reference to the previous value. The previous value is stored in the T-record before the compare and swap is performed (lines 48 49) with a failed compare and swap resulting in the abort of the transaction. If while performing these compare and swaps the transaction notices that another LIVE transaction is updating this memory, it aborts itself (line 48). By using these T-records instead of locks concurrent operations have access to necessary metadata used to ensure correctness.

The operation then advances the $G V C$, taking the new value of the clock as the logical time for this transaction (line 54). Following this, the read set of the transaction is validated for correctness (line 54). Once validation has been performed the operation must ensure that non of its writes have been concurrently overwritten by non-transactional operations (lines 55, 58) if so then the transaction must abort in order to (line 57) to ensure consistency. During this check the transaction updates the time value of its T-records to the transactions logical time (line 56) similar to the way TL2 stores time values in the lock array so that future operations will know the serialization of this transaction's updates.

Finally the transaction can mark its updates as valid by changing its status variable from LIVE to COMMITTED (line 59). This is done using a compare and swap as there could be a concurrent non-transactional operations 
trying to abort the transaction. If this succeeds then the transaction has successfully committed, otherwise it must abort and restart.

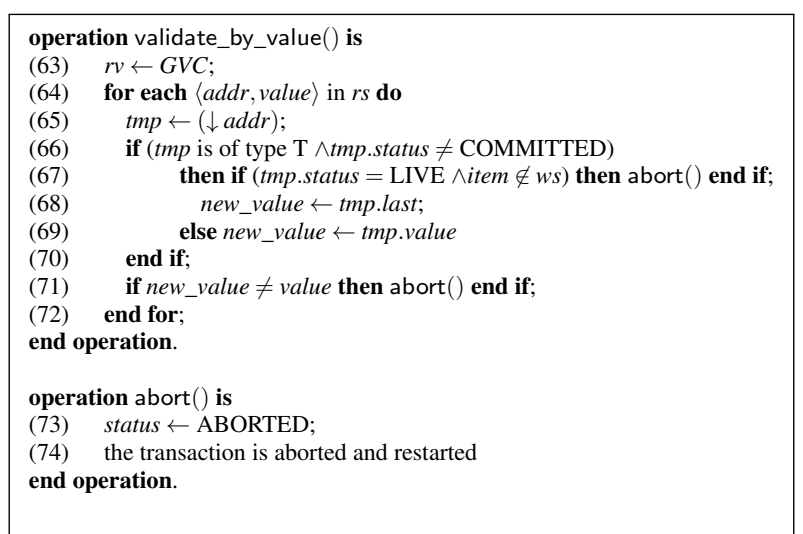

Figure 6: Transactional helper operations.

Transactional Helping Operations. Apart from the basic operations for starting, committing, reading and writing, a transaction makes use of helper operations to perform aborts and validate the read set. Pseudo-code for this kind of helper operations is given in Fig. 6

Operation validate_by_value() is an operation that performs validation of the read set of a transaction. Validation fails if any location in $r s$ is currently being updated by another transaction (line 67) or has had its changed since it was first read by the transaction (line 71) otherwise it succeeds. The transaction is immediately aborted if validation fails (lines 67, 71,. Before the validation is performed the local variable $r v$ is updated to be the current value of GVC (line 63). This is done because if validation succeeds then transaction is valid at this time with a larger clock value possibly preventing future validations and aborts.

When a transaction is aborted in the present algorithm, the status of the current transaction is set to ABORTED (line 73 and it is immediately restarted as a new transaction.

\section{Conclusion}

This paper has presented an algorithm that achieves non-blocking strong isolation "on top of" a TM algorithm based on logical dates and locks, namely TL2. In the case of a conflict between a transactional and a nontransactional operation, this algorithm gives priority to the non-transactional operation, with the reasoning that while an eventual abort or restart is part of the specification of a transaction, this is not the case for a single shared read or write operation. Due to this priority mechanism, the proposed algorithm is particularly appropriate for environments in which processes do not rely heavily on the use of especially large transactions along with nontransactional write operations. In such environments, terminating strong isolation is provided for transactions, while conventional read and write operations execute with a small additional overhead.

\section{References}

[1] Afek, Y., Avni, H., Dice, D., Shavit, N.: Efficient lock free privatization. In: Proc. 14th Int'l conference on Principles of Distributed Systems (OPODIS'10), pp. 333-347, Springer-Verlag, LNCS \#6490 (2010)

[2] Crain, T., Imbs, D., Raynal, M.: Towards a Universal Construction for Transaction-based Multiprocess Programs. In: Proc. 13th Int'l Conference on Distributed Computing and Networking (ICDCN'12), pp. 61-75, Springer Verlag LNCS \#7129 (2012)

[3] Dalessandro, L., Scott, M.: Strong Isolation is a Weak Idea. In: Proc. Workshop on transactional memory (TRANSACT'09) (2009) 
[4] Dice, D., Matveev, A., Shavit, N.: Implicit privatization using private transactions. In: Proc. Workshop on transactional memory (TRANSACT'10) (2010)

[5] Dice, D., Shalev O., Shavit, N.: Transactional Locking II. In: Proc. 20th Int'l Symp. on Distributed Computing (DISC'06), Springer-Verlag, LNCS \#4167, pp. 194-208 (2006)

[6] Guerraoui, R., Kapalka, M.: On the correctness of transactional memory. In: Proc. 13th ACM SIGPLAN Symposium on Principles and Practice of Parallel Programming (PPoPP '08), ACM Press, pp. 175-184 (2008)

[7] Harris, T., Larus, J., Rajwar, R.: Transactional Memory, 2nd edition, Synthesis Lectures on Computer Architecture, Morgan \& Claypool Publishers (2006)

[8] Herlihy, M., Moss J.M.B.: Transactional memory: architectural support for lock-free data structures, In: Proc. of the 20th annual Int'l Symposium on Computer Architecture (ISCA '93), ACM Press, pp. 289-300 (1993)

[9] Imbs, D., Raynal, M.: A versatile STM protocol with invisible read operations that satisfies the virtual world consistency condition. In: 16th Colloquium on Structural Information and Communication Complexity (SIROCCO’09), Springer Verlag LNCS \#5869, pp. 266-280 (2009)

[10] Maessen, J.-W., Arvind, M.: Store Atomicity for Transactional Memory. Electronic Notes on Theoretical Computer Science, 174(9):117-137 (2007).

[11] Martin, M., Blundell, C., Lewis, E.: Subtleties of Transactional Memory Atomicity Semantics. IEEE Computer Architecture Letters, 5(2): (2006)

[12] Matveev, A., Shavit, N.: Towards a Fully Pessimistic STM Model. In: Proc. Workshop on transactional memory (TRANSACT'12) (2012)

[13] Minh, C., Trautmann, M., Chung, J., McDonald, A., Bronson, N., Casper, J., Kozyrakis, C., Olukotun, K.: An effective hybrid transactional memory system with strong isolation guarantees. In: SIGARCH Comput. Archit. News, 35(2):69-80 (2007)

[14] Papadimitriou, Ch.H.: The Serializability of Concurrent Updates. Journal of the ACM, 26(4):631-653 (1979)

[15] Schneider, F., Menon, V., Shpeisman, T., Adl-Tabatabai, A.: Dynamic optimization for efficient strong atomicity. In: ACM SIGPLAN Noticers, 43(10):181-194 (2008)

[16] Scott, M.L., Spear, M.F., Dalessandro, L., Marathe, V.J.: Delaunay Triangulation with Transactions and Barriers. In: Proc. 10th IEEE Int'l Symposium on Workload Characterization (IISWC '07), IEEE Computer Society, pp. 107-113 (2007)

[17] Shavit, N., Touitou, D.: Software transactional memory. Software Transactional Memory. In: Distributed Computing, 10(2):99-116 (1997)

[18] Shpeisman, T., Menon, V., Adl-Tabatabai, A.R., Balensiefer, S., Grossman, D., Hudson, R.L., Moore, K.F., Saha, B.: Enforcing isolation and ordering in STM. In: ACM SIGPLAN Noticers, 42(6):78-88 (2007)

[19] Spear M.F., Dalessandro L., Marathe V.J., Scott, M.L.: Ordering-Based Semantics for Software Transactional Memory. In: Proc 12th Int'l Conf. on Principles of Distributed Systems (OPODIS '08), Springer-Verlag LNCS \#5401, pp. 275-294 (2008)

[20] Spear, M.F., Marathe, V.J., Dalessandro, L., Scott, M.L.: Privatization techniques for software transactional memory. In: Proc. 26th annual ACM symposium on Principles of Distributed Computing (PODC '07), . ACM press, pp. 338-339, (2007) 


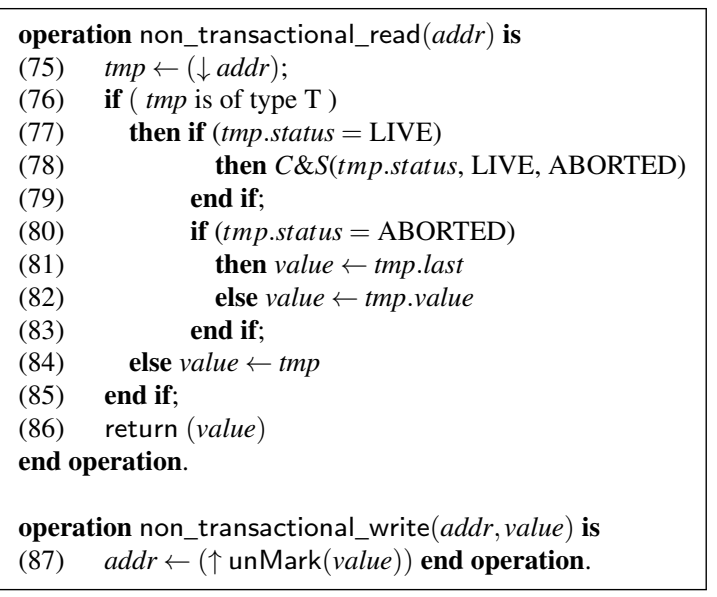

Figure 7: Non-transactional operations for reading and writing a variable.

\section{A Version of algorithm that does not use NT-records}

This algorithm also provides wait-free NT read and write operations. The difference is that NT-records are not used. Instead NT values are read and written directly from memory. By doing this, memory allocations are not needed in NT writes and NT reads have one less level of indirection.

The cost of this is more frequent validations required in transactions when conflicts with NT writes occur. This algorithm is shown in Figs. 7, 9

\section{B Version of algorithm with non-blocking NT-reads and blocking NT- writes}

This algorithm allows wait-free NT read operations. The only change that is needed to the base TL2 algorithm is that when an item is locked it points to the write-set of the transaction, and that each transaction has a marker that is initialized as LIVE and is set to COMMITTED just before the transaction starts performing write backs during the commit phase. The NT-read operation is shown in Fig. 10. 


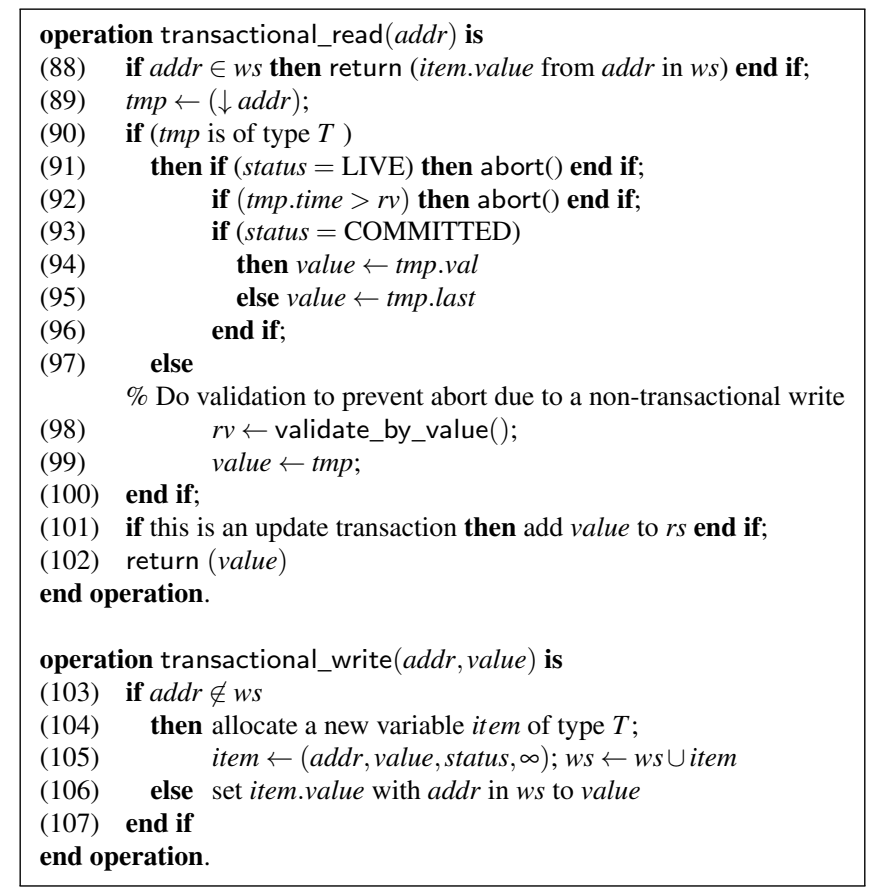

Figure 8: Transactional operations for reading and writing a variable.

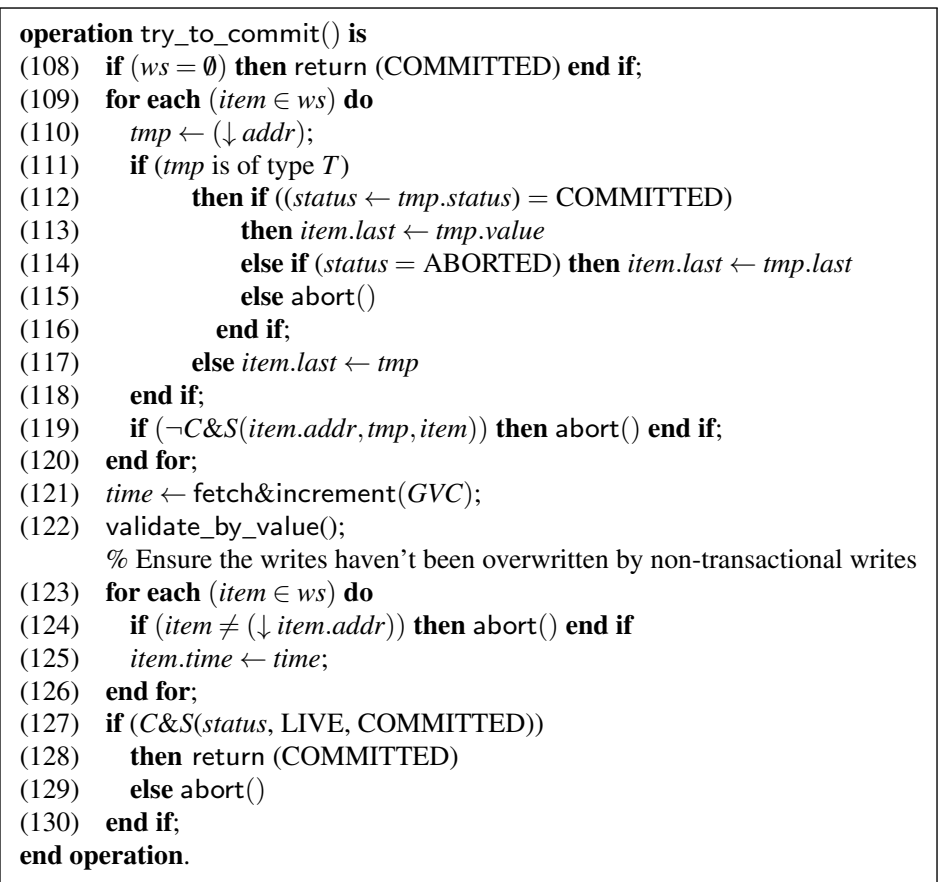

Figure 9: Transaction commit. 


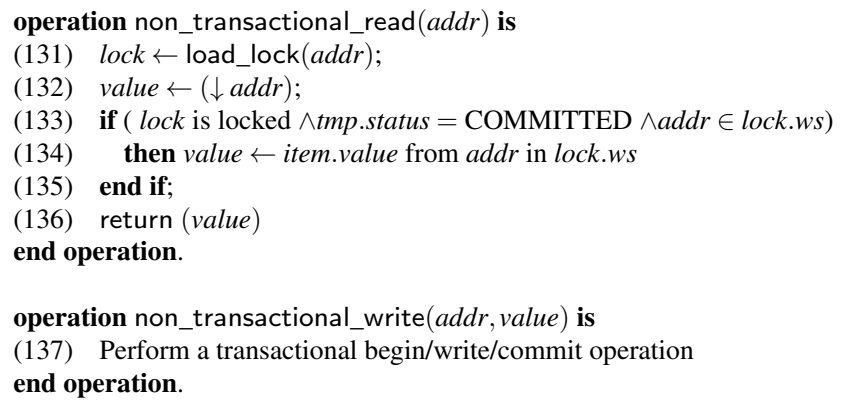

Figure 10: Non-transactional operations for reading and writing a variable. 


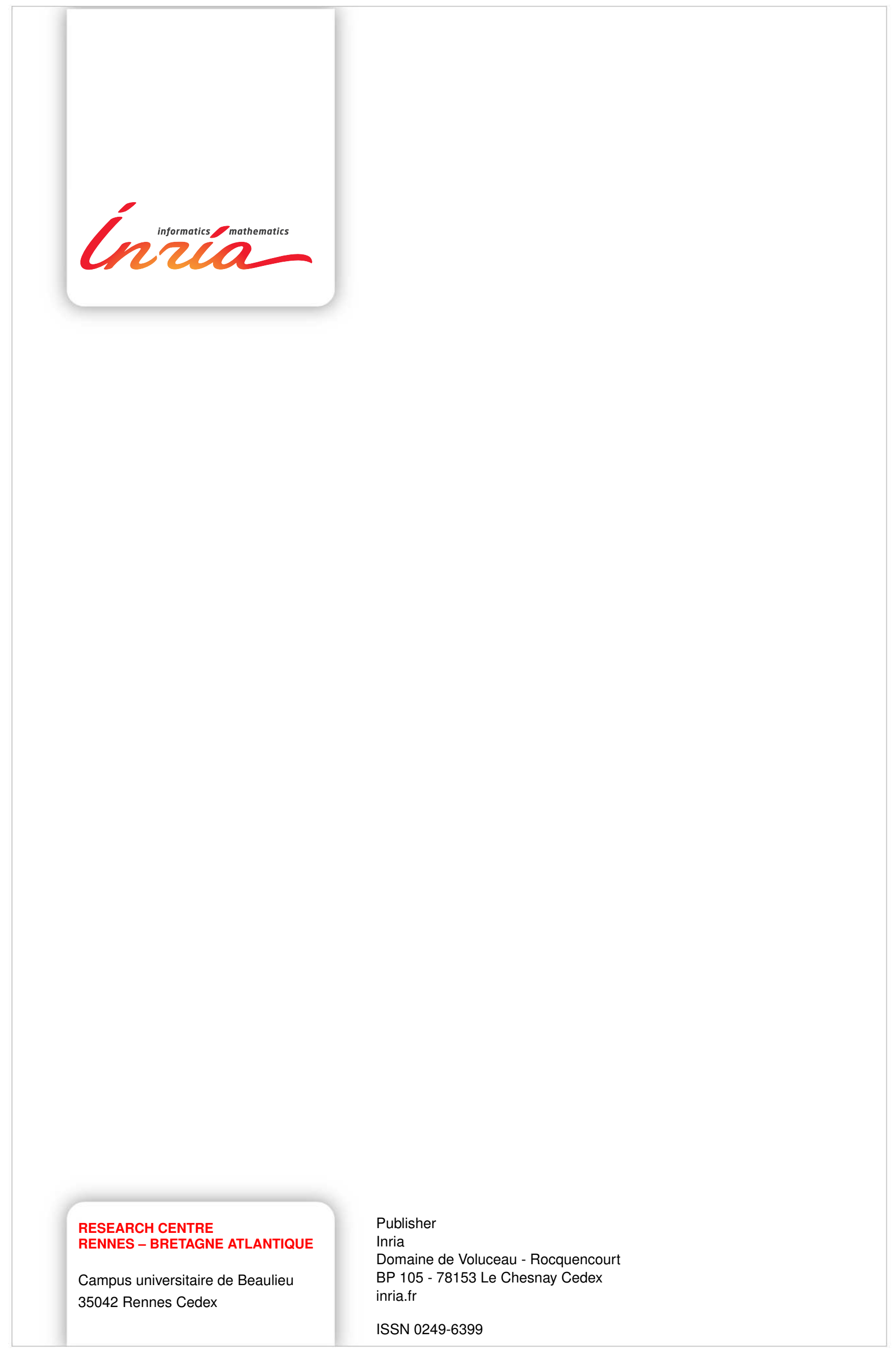

